\title{
Is G84.0+0.8 a high mass star formation site near the edge of the Pelican nebula?^
}

\author{
F. Comerón ${ }^{1}$, A. Pasquali ${ }^{2}$, and J. Torra ${ }^{3}$ \\ ${ }^{1}$ European Southern Observatory, Karl-Schwarzschild-Strasse 2, 85748 Garching, Germany \\ e-mail: fcomeron@eso.org \\ 2 Institute of Astronomy, ETH Hoenggerberg, 8093 Zurich, Switzerland \\ e-mail: pasquali@phys.ethz.ch \\ 3 Departament d'Astronomia i Meteorologia, Universitat de Barcelona, 08028 Barcelona, Spain \\ e-mail: jordi@am.ub.es
}

Received 28 October 2004 / Accepted 4 May 2005

\begin{abstract}
We present visible and near-infrared observations of the G84.0+0.8 HII region, a bright compact knot projected within the boundaries of the W80 complex dominated by the North America and Pelican nebulae. The spectrum of the nebula indicates a temperature of the ionizing stellar spectrum $T_{*} \simeq 40000-45000 \mathrm{~K}$ (corresponding to a O7-O5 star) and a density of the HII region $n \simeq 460 \mathrm{~cm}^{-3}$, with a foreground extinction of $A_{\mathrm{V}} \simeq 5.9 \mathrm{mag}$. A comparison of narrow-band near-infrared images through the $\mathrm{Br} \gamma$ and the $\mathrm{H}_{2} S(1) v=1 \rightarrow 0$ filters shows that G84.0+0.8 consists of a fan-shaped cavity in a molecular cloud at least partly bounded by a photodissociation region, filled with $\mathrm{Br} \gamma$-emitting ionized gas, and with a compact cluster at the tip of the fan. The brightest star at the position of the cluster is found to be a late G-type interloper. While membership of G84.0+0.8 in the local arm is well established from existing radial velocity measurements of the ionized gas, we find that the ionizing flux estimated from the size and density of the nebula on the one hand, and the radio continuum properties of the nebula on the other hand, are well below the expected ionizing flux of a mid, or even late, O-type star. We consider the possibility that G84.0+0.8 might be externally ionized by a nearby mid-O star. Currently available observations do not definitely confirm or reject the membership of G84.0+0.8 in the W80 complex, although a larger distance seems favored by the available data. Nevertheless, we can firmly rule out the possibility that it represents a massive star forming site in that complex, as its appearance as a compact HII region containing an embedded cluster may lead one to think.
\end{abstract}

Key words. HII regions - ISM: individual objects: G84.0+0.8 - ISM: individual objects: W80

\section{Introduction}

W80 (Westerhout 1958) is one of the nearest extended complexes of ionized gas, encompassing the well-known North America and Pelican nebulae (NGC 7000 and IC 5070 respectively). Despite being at a distance not much larger than the Orion Nebula (600 pc vs. 450 pc; Laugalys \& Straizys 2002), the differences between both complexes are striking. W80 is much more diffuse, it extends over a diameter of $\simeq 3^{\circ}(\simeq 32 \mathrm{pc})$, and it does not possess any analog of the intense massive star forming activity of the Trapezium cluster. The main responsible for the ionization of the W80 complex is most likely an isolated O5V star, J205551.3+435225, recently identified by Comerón \& Pasquali (2005). Signs of recent and ongoing star formation at low and intermediate masses have been identified

* Based on observations collected at the Centro Astronómico Hispano-Alemán (CAHA) at Calar Alto, operated jointly by the Max-Planck Institut für Astronomie and the Instituto de Astrofísica de Andalucía (CSIC). in several areas of W80, particularly in the dark cloud L935 that separates the North America and the Pelican nebulae (Herbig 1958) and in the interface between L935 and the Pelican nebula (Bally \& Reipurth 2003).

Visible images of the region (Fig. 1) show the compact, bright knot G84.0+0.8 to the West of the Pelican nebula at $\alpha(2000)=20^{\mathrm{h}} 45^{\mathrm{m}} 38^{\mathrm{s}} 9, \delta(2000)=+44^{\circ} 15^{\prime} 09^{\prime \prime}$. The knot is identified by Wendker et al. (1991) as the thermal radio source ECX6-38. Comerón \& Torra (2001) reported the existence of a compact cluster clearly visible in near-infrared images, also noted by Dutra \& Bica (2001) in 2MASS images. Its location in the sky close to W80, and the possibility that it may be one of the few locations where massive star formation in that complex has taken place in the most recent past, seem to deserve some detailed consideration. In this paper we present additional observations that provide further insight into the nature of G84.0+0.8, its possible relationship to the W80 complex and, more generally, to the much more extended Cygnus $\mathrm{X}$ region in which both W80 and G84.0+0.8 lie. 


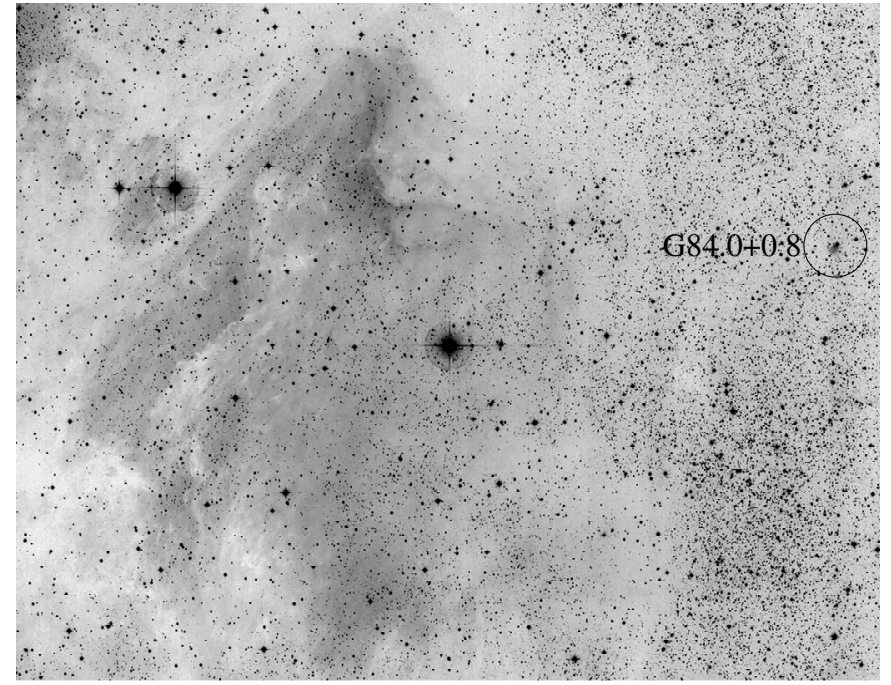

Fig. 1. The region of the Pelican nebula (IC 5070), including the compact HII region G84.0+0.8 discussed in this paper. The image covers a field of $107^{\prime} 8 \times 84^{\prime} 1$ and is a negative print of an image obtained with the Schmidt telescope on Calar Alto, courtesy of the Centro Astronómico Hispano-Alemán.

\section{Observations}

The dataset presented here is composed of near-infrared narrow-band imaging and spectroscopy in the visible obtained with the $2.2 \mathrm{~m}$ telescope at the Calar Alto Observatory. The near-infrared images were obtained on the night of 13/14 June 2001 using MAGIC, a NICMOS3-based infrared camera and spectrograph. The $0^{\prime \prime} 64 \mathrm{pix}^{-1}$ scale at that telescope provides a field of view of $2^{\prime} 7 \times 2^{\prime} 7$ on the $256 \times 256$ array. We obtained images through the Brackett $\gamma(2.166 \mu \mathrm{m})$ and $\mathrm{H}_{2} \mathrm{~S}(1) v=1 \rightarrow 0(2.122 \mu \mathrm{m})$ filters. Each narrow-band image has a total exposure time of $9 \mathrm{~min}$ split in 9 dithered 1-min pointings. Due to the size of the dither pattern the field of view covered in each frame is $3^{\prime} 05 \times 3^{\prime} 05$.

Visible spectroscopy was obtained in two separate runs, the first one on the nights of 28/29 and 29/30 October 2001 in Service Mode, using Director's Discretionary Time, and the second one on the night of 2/3 August 2004. We used CAFOS, the facility's visible imager and spectrograph, with the $\mathrm{B} 200$ grating that provided a resolution $R=\lambda / \Delta \lambda \simeq 375$ at $\lambda=5000 \AA$ with the $1^{\prime \prime} 5$ slit used and covered the entire visible range. The slit was placed on each of the four brightest stars of the cluster identified in the visible, and thus provided cuts across the entire HII region in the North-South direction along which the slit was oriented. Each star was observed at least twice, with exposure times between 10 and 15 min per observation. Wavelength calibration was achieved by taking short exposures of three lamps of $\mathrm{HgCd}, \mathrm{He}$, and $\mathrm{Rb}$. Relative flux calibration was performed only on the observations obtained in August 2004 by observing the spectrophotometric standard G191-B2B.
The reduction of all observations was carried out using standard tasks and dedicated scripts under IRAF ${ }^{1}$.

\section{The G84.0+0.8 region and its environment}

In large-scale molecular line maps of the Cygnus $\mathrm{X}$ complex the location of G84.0+0.8 coincides with a peak in the column density of molecular gas (Leung \& Thaddeus 1992). ${ }^{12} \mathrm{CO}$ velocity-channel maps in the $-5 \mathrm{~km} \mathrm{~s}^{-1}<v<$ $-2.5 \mathrm{~km} \mathrm{~s}^{-1}$ range show that this peak lies on the rim of a large elliptical structure having approximate dimensions of $5^{\circ} 5 \times$ $2^{\circ} 9$, containing also W80. Molecular emission inside the ellipse is detected at less negative and small positive velocities, particularly in the $0 \mathrm{~km} \mathrm{~s}^{-1}<v<2.5 \mathrm{~km} \mathrm{~s}^{-1}$ interval, which might lead to the interpretation of the entire region as an expanding hemispherical shell with the near hemisphere missing. The radial velocity of G84.0+0.8, measured from the $\mathrm{H} 110 \alpha$ hydrogen radio recombination line, is $v=-6.6 \mathrm{~km} \mathrm{~s}^{-1}$ (Piepenbrink \& Wendker 1988), close to the velocity of the peak ${ }^{12} \mathrm{CO}$ emission. This definitely places G84.0+0.8 as a member of the local arm rather than of the Perseus arm, characterized by negative velocities of $-30 /-40 \mathrm{~km} \mathrm{~s}^{-1}$ in that direction, but the fact that the local arm is seen approximately end-on, and the low sensitivity of radial velocities to the distance in the direction of Cygnus within a few kiloparsecs from the Sun, leaves room for a large uncertainty as to the location of G84.0+0.8 along the line of sight, as well as for most members of the local arm in the same direction (Odenwald \& Schwartz 1993).

Mid-infrared images obtained by the IRAS and MSX satellites show G84.0+0.8 as a small, isolated and moderately elongated source. The IRAS fluxes at 12, 25, 60 and $100 \mu \mathrm{m}$ are respectively $(147 \pm 15),(390 \pm 15),(3000 \pm 150)$, and $(3290 \pm$ 750) Jy. A comparison of these fluxes to the catalog of compact HII regions of Chini et al. (1987) indicates a luminosity $L\left(L_{\odot}\right)=7.7 \times 10^{3} \mathrm{D}(\mathrm{kpc})^{2}$. The comparison is based on the sources with the bluest IRAS colors in that catalog, whose spectral energy distributions are the ones most closely resembling that of G84.0+0.8. However, the mid-infrared spectral energy distribution of G84.0+0.8 is bluer than that of any of the sources in Chini et al.'s (1987) catalog, as will be discussed in Sect. 5 .

\subsection{Infrared imaging of $G 84.0+0.8$}

The two narrow-band infrared images presented in Fig. 2 show the distribution of the ionized gas $(2.166 \mu \mathrm{m}$ image $)$ and of the photodissociation region $(2.122 \mu \mathrm{m}$ image $)$ produced by ultraviolet but non-ionizing radiation penetrating the surrounding molecular gas. The associated cluster is clearly seen as well. Additional broad-band images of G84.0+0.8 can be found in Comerón \& Torra (2001). The $2.122 \mu \mathrm{m}$ image shows that the contours of the cavity filled by the $\mathrm{Br} \gamma$-emitting gas are surrounded by a photodissociation region, but that most of the

1 IRAF is distributed by NOAO, which is operated by the Association of Universities for Research in Astronomy, Inc., under contract to the National Science Foundation. 


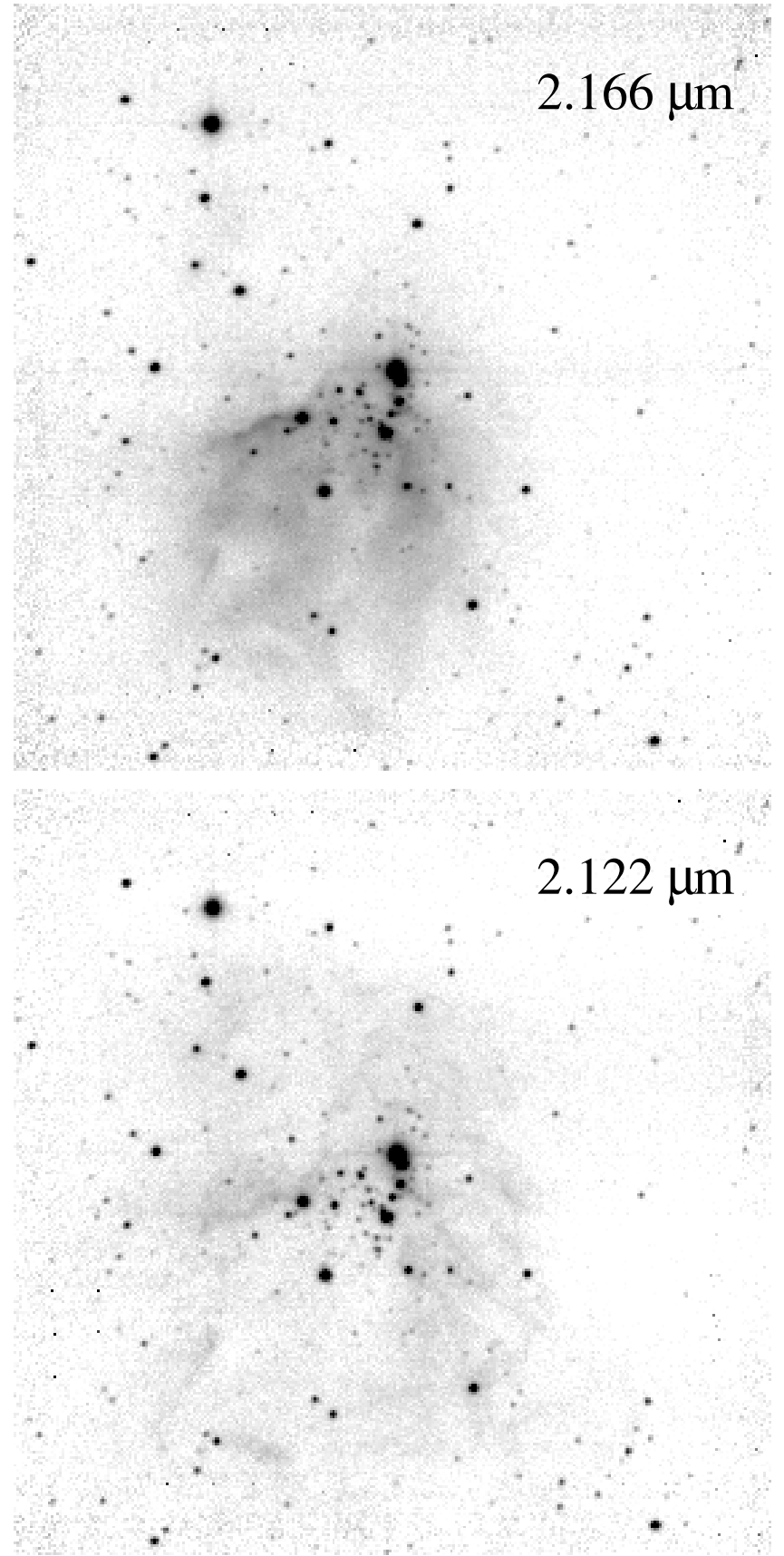

Fig. 2. Narrow-band infrared images of G84.0+0.8 showing the distribution of ionized hydrogen (top) and excited molecular hydrogen (bottom), outlining the overall structure of a cavity in the molecular cloud filled with ionized gas. The embedded cluster is clearly seen in both images. North is at the top and East to the left. The size of both images is $3^{\prime} 05 \times 3^{\prime} 05$.

$\mathrm{H}_{2}$ emission actually arises from the North-Northeastern half of the region, on which part of the cluster is projected.

\subsection{Visible spectroscopy and physical conditions of the nebula}

Based on the information available at the time of their study, Comerón \& Torra (2001) proposed that G84.0+0.8 is a compact HII region belonging to the North America/Pelican

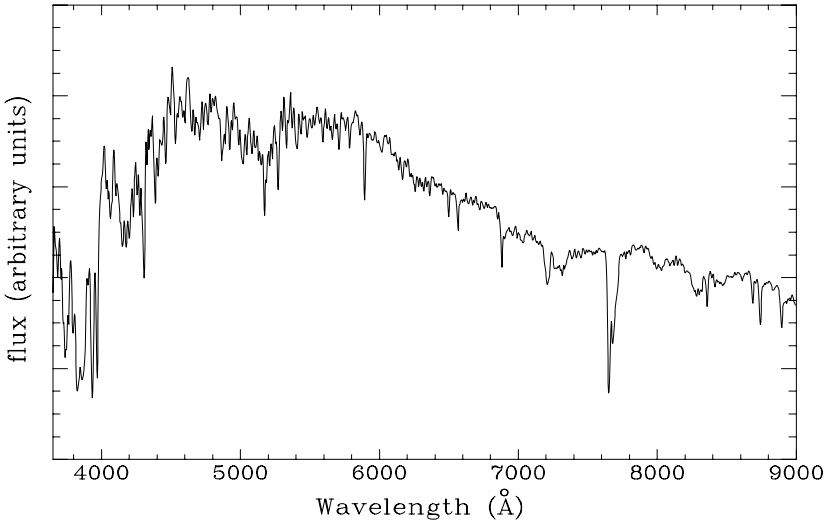

Fig. 3. Spectrum of the brightest star that appears projected on the embedded cluster, which we classify as a late-type giant and is thus unrelated to the cluster.

complex, ionized by a relatively late (B2V) star having a modest output of ionizing radiation. They tentatively identified the brightest star that appears projected on the cluster as the possible ionizing source.

The new spectroscopy suggests a fairly different picture. The star proposed by Comerón \& Torra (2001) as the ionizing candidate is clearly discarded as such by its spectrum (Fig. 3), which we classify as a late G-type giant by comparison to the atlas of low-resolution stellar spectra of Jacoby et al. (1984). The moderate extinction indicated by the colors provided by Comerón \& Torra (2001) is not as high as the one in the nebula as derived from its Balmer lines, suggesting that the $G$ star is in the foreground relative to G84.0+0.8 but is already partly obscured by dust, probably associated with the W80 complex.

The spectrum of the nebula, presented in Fig. 4, clearly shows HeI emission lines at $5876 \AA$ and $6678 \AA$ and a large $[\mathrm{OIII}] / \mathrm{H} \beta$ intensity ratio, indicating that the ionizing star must be of $\mathrm{O}$ type. We examined spectra obtained at several positions of the nebula sampled by the different slit positions, and did not find any significant changes among the line ratios that would indicate substantial variations in the physical conditions across the nebula. The density-sensitive [SII] $(6717 \AA) /[\mathrm{SII}](6731 \AA)$ ratio remains remarkably constant across the entire area of the nebula at a value indicative of a density in the $\sim 400-500 \mathrm{~cm}^{-3}$ range (see below). It does not show any noticeable change near the center, where the line of sight should probe denser gas if the nebula had an outwarddecreasing density distribution. In such case, the intrinsically lower $[$ SII $](6717 \AA) /[S I I](6731 \AA)$ ratio contributed by the denser regions to the integrated emission along the line of sight should cause a decrease in the $[\mathrm{SII}](6717 \AA) /[\mathrm{SII}](6731 \AA)$ ratio that is not observed. Moreover, the brightness distribution in the $\mathrm{Br} \gamma$ image (Fig. 2) shows only moderate fluctuations across the area occupied by the ionized gas, excluding the presence of much denser clumps not sampled by our slit settings. We are thus confident that the physical conditions derived from the spectrum presented in Fig. 4 can be considered as representative of the entire ionized nebula.

We have measured the integrated fluxes of the identified $\mathrm{H}, \mathrm{He}, \mathrm{O}$ and $\mathrm{S}$ lines with the purpose of computing the 


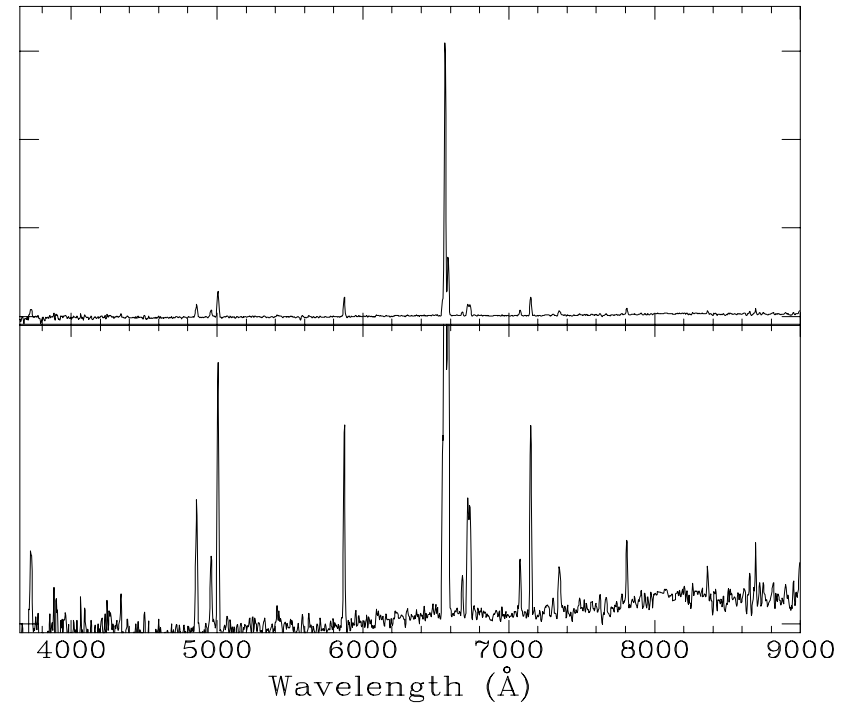

Fig. 4. Spectrum of the HII region, with the vertical scale increased in the bottom plot to enhance visibility of the faintest features. The prominence of the [OIII] lines near $\lambda=5000 \AA$ with respect to the adjacent $\mathrm{H} \beta$ and the strength of the $\mathrm{HeI}$ lines show that the nebula is ionized by a mid O-type star. The electron density inferred from the relative strengths of the [SII] doublet near $\lambda=6720 \AA$ is $460 \mathrm{~cm}^{-3}$, and the extinction from the $\mathrm{H} \beta / \mathrm{H} \alpha$ ratio is $A_{\mathrm{V}} \simeq 5.9$.

chemical composition of the nebula. From the $\mathrm{H} \alpha / \mathrm{H} \beta$ ratio in case B recombination theory (Osterbrock 1989), we derive an overall reddening $c_{\beta}$ of 2.21 . The extinction towards the nebula can also be calculated from the comparison between the observed $\mathrm{H} \beta / \mathrm{H} \alpha$ ratio and the intrinsic value expected from Case $\mathrm{B}$, adopting an extinction ratio $A(\mathrm{H} \beta) / A(\mathrm{H} \alpha)=1.424$ derived from the extinction law of Cardelli et al. (1989), from which we obtain $A_{\mathrm{V}}=5.9$. All line fluxes were corrected for reddening using the above $c_{\beta}=2.21$. Since neither the [NII] $\lambda 5755$ line nor the [OIII] $\lambda 4363$ emission are detected in the nebular spectrum, we have assumed an electronic temperature $T_{\mathrm{e}}$ of $8000 \mathrm{~K}$, which is the average value among Galactic HII regions (cf. Shaver et al. 1983). The [SII] $(6717 \AA) /[\mathrm{SII}](6731 \AA)$ flux ratio of G84.0+0.8 indicates an electron density $n_{\mathrm{e}}=460 \pm 100 \mathrm{~cm}^{-3}$, where the main contribution to the uncertainty is due to variations in the line ratios across the parts of the nebula sampled by the spectrograph slit. We have then computed the ionic abundance of each element from the line dereddened fluxes using the IRAF NEBULAR package and imposing $T_{\mathrm{e}}=8000 \mathrm{~K}$ and $n_{\mathrm{e}}=460 \mathrm{~cm}^{-3}$. The ionic abundances of the same ion from different emission lines were subsequently weighted by the flux of the corresponding line and averaged together. The results, which are summarized in Table 1, are in good agreement with the abundances derived for Galactic HII regions (Shaver et al. 1983).

Together with the density derived above, the $[\mathrm{OIII}] / \mathrm{H} \beta \simeq 2$ flux ratio and the strength of the HeI lines are both consistent in firmly constraining the temperature of the ionizing star to a value above $40000 \mathrm{~K}$. A comparison to the calculated spectrum of model nebulae ionized by a single star (Stasińska \& Schaerer 1997 ) indicate a temperature in the range between $40000 \mathrm{~K}$ and $45000 \mathrm{~K}$ (the upper limit being less strongly constrained),
Table 1. Ionic abundances in G84.0+0.8.

\begin{tabular}{lc}
\hline \hline Ion & $12+\log (\mathrm{X} / \mathrm{H})$ \\
\hline $\mathrm{He}^{+}$ & 0.11 \\
$\mathrm{~N}^{+}$ & 7.40 \\
$\mathrm{O}^{+}$ & 8.90 \\
$\mathrm{O}^{++}$ & 8.10 \\
$\mathrm{~S}^{+}$ & 6.12 \\
\hline
\end{tabular}

corresponding to a spectral type between O7 and O5 (Schaerer $\&$ de Koter 1997). An ionizing spectral type later than O7 can thus be confidently ruled out by the spectrum of the nebula.

\section{4. lonizing G84.0+0.8}

Assuming that G84.0+0.8 is roughly spherical and ionizationbounded, and neglecting absorption of ultraviolet radiation by dust inside the HII region, the input rate of ionizing photons $Q_{0}$ required to keep the nebula ionized should be

$Q_{0}=\frac{4 \pi}{3} \beta_{2} n_{0}^{2} R^{3}$

where $\beta_{2}$ is the recombination rate of protons and electrons excluding direct recombinations to the ground level. The value of $Q_{0}$ derived in this way is rather uncertain, as it explicitly depends on the geometry and size of the region and its density structure. A more direct measurement of $Q_{0}$ is provided by the radio continuum flux, which for an optically thin HII region at a distance $D$ is given by

$Q_{0}\left(\mathrm{~s}^{-1}\right)=1.8 \times 10^{46} D(\mathrm{kpc})^{2} v(\mathrm{GHz})^{0.1} S_{v}(\mathrm{Jy})$

(Altenhoff et al. 1960). The radio continuum spectrum of G84.0+0.8 is indeed characteristic of an optically thin HII region, with a flux density $S_{v}$ varying only mildly with frequency. Published measurements are $2420 \mathrm{mJy}$ at $5 \mathrm{GHz}$ (Gregory \& Taylor 1986; a similar value, $2574 \mathrm{mJy}$, is given by Griffith et al. 1991), and $2459 \mathrm{mJy}$ at $1.42 \mathrm{GHz}$ (Normandeau et al. 1992). Measurements between $408 \mathrm{MHz}$ and $5 \mathrm{GHz}$ published by Wendker et al. (1991) are greater by approximately $20 \%$, but also have a very slight frequency dependency showing that G84.0+0.8 is optically thin down to at least $408 \mathrm{MHz}$.

A comparison with computed $Q_{0}$ values for model $\mathrm{O}$ stars (Schaerer \& de Koter 1997) shows that the values calculated from Eqs. (1) and (2) if G84.0+0.8 were at the distance of W80 $(D=600 \mathrm{pc}$, implying $R \simeq 0.08 \mathrm{pc}$ ) are respectively $3.4 \times$ $10^{45} \mathrm{~s}^{-1}$ and $1.8 \times 10^{46} \mathrm{~s}^{-1}$, between three and four orders of magnitude below the ionizing flux provided by a mainsequence star with the lowest temperature consistent with the spectrum of the HII region, $T_{*}=40000 \mathrm{~K}$ (spectral type O7V), which is $Q_{0}=1.1 \times 10^{49} \mathrm{~s}^{-1}$. The result is not significantly changed if other sets of models are used (e.g. Vacca et al. 1996, and the discussion in Schaerer \& de Koter 1997). Absorption by dust of most of the ionizing radiation is an unviable explanation, given the required fraction of only one ultraviolet photon available for hydrogen ionization for each 1000-10000 photons absorbed by dust, which is at least two orders of magnitude below the most extreme estimates made in ultracompact HII regions (Wood \& Churchwell 1989). Moreover, both 


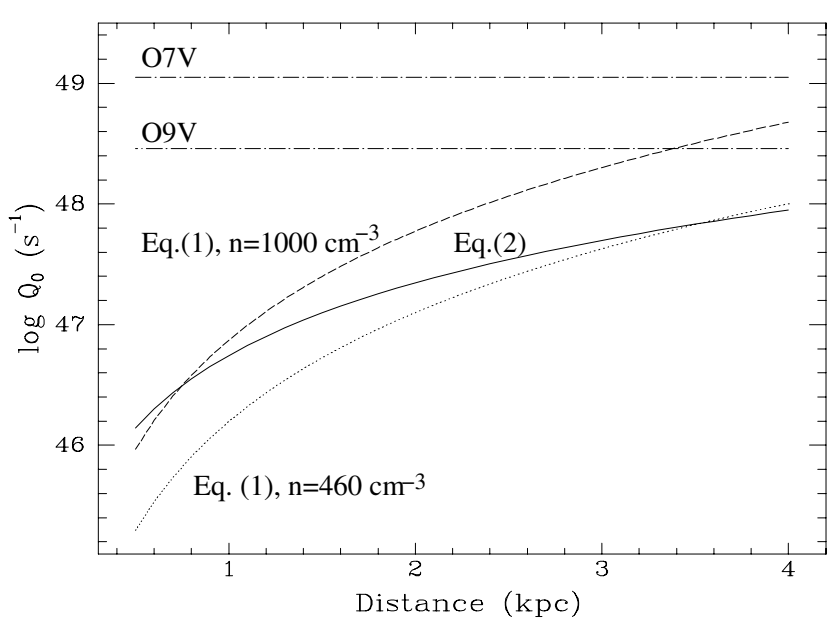

Fig. 5. The ionizing flux of G84.0+0.8 as inferred from its radio continuum flux (Eq. (2), solid line); and from its angular size and density using respectively $n=460 \mathrm{~cm}^{-3}$ (Eq. (1); dashed line) and $n=1000 \mathrm{~cm}^{-3}$ (dotted line). The ionizing fluxes produced by $\mathrm{O} 7 \mathrm{~V}$ and $\mathrm{O} 9 \mathrm{~V}$ stars, whose temperatures are respectively $T_{*}=41000 \mathrm{~K}$ and $T_{*}=35900 \mathrm{~K}$ (Schaerer \& de Koter 1997) are given for reference. As noted in the text, both $T_{*}=35900 \mathrm{~K}$ and $n=1000 \mathrm{~cm}^{-3}$ are excluded by the spectroscopy of the nebula, and are plotted here for reference only.

the moderate extinction and the modest IRAS fluxes argue against a significant amount of reprocessing of star light by dust: at a distance of $600 \mathrm{pc}$, the integrated infrared luminosity $L_{\mathrm{IR}} \simeq 2800 L_{\odot}$ is about two orders of magnitude below the bolometric luminosity of a O7V star. We also consider it unlikely that the HII region is largely density-bounded, given the direct evidence that the $2.122 \mu \mathrm{m}$ image provides for a large reservoir of molecular gas around most of the nebula, which must cover a sizeable solid angle of the sky as seen from any star in the cluster.

The discrepancy is reduced if G84.0+0.8 is actually farther than W80, a reasonable possibility given the wide range of distances estimated for individual regions in the direction of Cygnus X (e.g. Odenwald \& Schwartz 1993; Behre et al. 2004). Figure 5 summarizes the magnitude of the disagreement between the ionizing flux yielded by the coolest star consistent with the nebular spectrum, and the ionizing flux inferred from the properties of the nebula as a function of the assumed distance. The latter is limited to $4 \mathrm{kpc}$, since higher values are excluded by the radial velocity. We have indicated in Fig. 5 the ionizing flux yielded by a O9V star $\left(T_{*}=35900 \mathrm{~K}\right.$; Schaerer $\&$ de Koter 1997), even though its temperature is insufficient to produce the [OIII] and HeI line intensities observed in the nebula. Likewise, we have also plotted the values yielded by Eq. (1) assuming a density $n=1000 \mathrm{~cm}^{-3}$, twice as high as that inferred from the [SII] line ratio. Such density would result in a $[\mathrm{SII}](6717 \AA) /[\mathrm{SII}](6731 \AA)$ ratio around 0.8 , whereas the observed value in our spectra is always above 1.0. The O9V and $n=1000 \mathrm{~cm}^{-3}$ cases can thus be regarded as illustrating the degree of the departures with respect to the observations that would have to be introduced in order to achieve a marginal agreement between inferred ionizing fluxes, rather than as limiting cases allowed by the observations.
The ionizing fluxes as derived from Eqs. (1) and (2) are coincident if the distance is $3.5 \mathrm{kpc}$, near the upper limit allowed by the radial velocity. The simplifying assumptions of a spherical nebula with uniform density entering Eq. (1), and the uncertainties in the measured radio continuum fluxes entering Eq. (2), prevent us from considering this as an accurate distance determination. However, the combination of Eqs. (1) and (2) does hint at a distance significantly greater than that of the W80 complex, although a significant discrepancy by a factor $\sim 15$ remains between the derived ionizing fluxes and that of a O7V star when a distance of $3.5 \mathrm{kpc}$ is adopted. The optically thin spectral energy distribution in the radio continuum even at $408 \mathrm{MHz}$ also argues for a shorter distance by implying a small optical depth, given that $\tau_{408 \mathrm{MHz}} \ll 1$ requires an emission measure $E M \ll 3 \times 10^{5} \mathrm{~cm}^{-6}$ pc (Mezger \& Henderson 1967). Since the electron density is well constrained by the spectrum of the nebula, a size along the line of sight not exceeding a few tenths of a parsec is needed to keep $E M$ small.

\section{The embedded cluster}

Figure 6 shows the 2MASS color-magnitude diagram of the stars appearing within $20^{\prime \prime}$ of the center of the cluster. The positions expected for $\mathrm{O} 7 \mathrm{~V}$ and $\mathrm{B} 2 \mathrm{~V}$ stars at $600 \mathrm{pc}$ reddened by different amounts are plotted with dashed lines, as well as the unreddened main sequence at that distance between $\mathrm{O} 5 \mathrm{~V}$ and F0V (using data compiled by Drilling \& Landolt 2000; and Tokunaga 2000). We also plot the position of an O7V star at $3.5 \mathrm{kpc}$ (see Sect. 4) reddened by varying amounts. The brightest object is the unrelated G-type star whose spectrum is presented in Fig. 3. The red star on the B2V locus at $600 \mathrm{pc}$ with $\left(H-K_{\mathrm{S}}\right)=1.49$ has a comparatively blue $(J-H)=1.39$, probably indicating substantial reprocessing by circumstellar material, and thus a significant non-photospheric contribution to the $K_{\mathrm{S}}$ flux.

Judging from their positions in the color-magnitude diagram the spectral types of the brightest stars would not be consistent with an O7V or earlier type unless G84.0+0.8 were at least $\sim 3.5 \mathrm{kpc}$ from the Sun, or unless such an O7V star were obscured by $A_{\mathrm{V}}>50 \mathrm{mag}$. The colors of the brightest members suggest visible extinctions in the $A_{\mathrm{V}}=4-12$ range, in agreement with the value derived from the Balmer lines of the HII region (Sect. 3.2). No photospheric features can be discerned in our visible spectra of these stars, preventing a spectroscopic determination of the distance of the region.

The 9 stars (excluding the G-type interloper) that lie on or above a reddening vector having its origin at the end of the solid, nearly vertical line in Fig. 6 allow us to obtain a rough estimate of the mass of the cluster if all these stars are actual members. Assuming a distance of $600 \mathrm{pc}$, a mass of $1.6 M_{\odot}$ for F0V stars (Drilling \& Landolt 2000), and a Miller-Scalo log-normal initial mass function (Miller \& Scalo 1979), we obtain a cluster mass of $\sim 50 M_{\odot}$. This is a very rough determination, probably affected by inaccurate photometry given the compactness of the cluster, close binarity, circumstellar emission, and small-numbers statistics in the sampling of the initial mass function. However, it suffices to characterize the cluster 


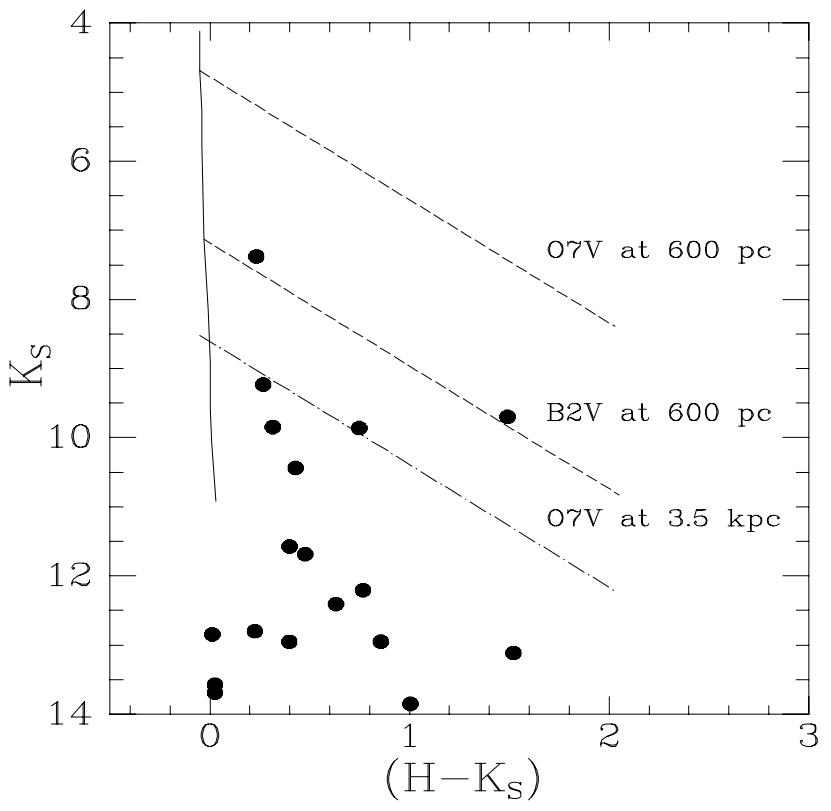

Fig. 6. 2MASS color-magnitude diagram containing the members of the cluster within a radius of $20^{\prime \prime}$ of $\alpha(2000)=20^{\mathrm{h}} 45^{\mathrm{m}} 38^{\mathrm{s}} 0$, $\delta(2000)=+44^{\circ} 15^{\prime} 20^{\prime \prime}$. The locus of the unreddened main sequence at $600 \mathrm{pc}$ and three selected extinction vectors (whose slope is given by the Rieke \& Lebofsky 1985, extinction law) are given, including one for the case of a O7V star at $3.5 \mathrm{kpc}$.

as a modest aggregate with no massive stars earlier than mid$\mathrm{B}$ type if it were a member of the W80 complex.

This picture changes dramatically if we assume a distance of $3.5 \mathrm{kpc}$. In that case there are two possible O7V stars in the cluster, and the limiting vector used above marks instead the locus of B2V stars with masses of $9.4 M_{\odot}$ (Drilling \& Landolt 2000). Using the same method described above, we now estimate a cluster mass of $\sim 870 M_{\odot}$, making it a tight massive cluster with a mass about 6 times that of the Trapezium occupying a similar volume (Herbig \& Terndrup 1986). The luminosity, dominated by the O-type members, is then $L \sim 7 \times 10^{5} L_{\odot}$. The luminosity reprocessed by dust and emitted at longer wavelengths, $\simeq 9.4 \times 10^{4} L_{\odot}$, is rather low as compared with that of other compact HII regions harboring O-type stars (Chini et al. 1987). Such compact HII regions display very similar spectral energy distributions in the IRAS bands, with a standard deviations from the average of only $25 \%$. However, the spectral energy distribution of G84.0+0.8 HII region is distinctly bluer than any of the objects in the catalog of Chini et al. (1987). As those authors note, the infrared spectral energy distributions shortwards of $12 \mu \mathrm{m}$ tend to be steeper for earlier types of the embedded stars, which is attributed to the action of their winds or their radiation pressure in clearing hot dust from their vicinity. The need for such dust-free cavities is a well established feature of models of compact and ultracompact HII regions, both theoretical and observationally (e.g. Faison et al. 1998; Inoue 2002, and references therein). The decreased efficiency of such dust removal mechanisms in cooler stars may explain the existence of larger amounts of hot dust in their immediate surroundings, whose higher temperatures increase the contribution to the fluxes at the shortest wavelengths. In this framework, the unusually blue mid-infrared spectral energy distribution of G84.0+0.8 may be taken as an indication of a lack of O-type stars, and would thus argue in favor of a distance significantly shorter than $3.5 \mathrm{kpc}$.

\section{Discussion}

In the previous sections we have stressed the problems encountered when trying to explain G84.0+0.8 as a simple, compact HII region ionized by its embedded cluster due to the large mismatch between the ionizing flux provided by a star of the temperature required by the spectrum of the HII region and the ionizing flux inferred from the visible/near infrared and the radio continuum properties of the nebula.

An intriguing possibility is that G84.0+0.8 might be ionized by stars external to the nebula, with G84.0+0.8 intercepting only a small fraction of their ultraviolet fluxes. In this picture, the energy output of the young stars of the embedded cluster would have blown a low-density cavity in their parental molecular cloud, exposing it to an ambient radiation field characterized by the spectral energy distribution of a mid O-type star, and by a photon flux high enough to ionize a relative large volume of the $n \simeq 460 \mathrm{~cm}^{-3}$ gas. Two known O-type members of W80 could in principle produce the nebular spectrum of G84.0+0.8. The first one is J205551.3+435225, the O5V star recently identified by Comerón \& Pasquali (2005) as the main star responsible for the ionization of the complex. Another suitable candidate is the O6V star HD 199579, which is projected on the North America nebula. These stars lie respectively 113' and $124^{\prime}$ from G84.0+0.8, corresponding to projected distances of $19.7 \mathrm{pc}$ and $21.6 \mathrm{pc}$ on the plane of the sky. An upper limit to the intercepted ionizing flux $Q_{0}^{\prime}$ from these stars at the position of $\mathrm{G} 84.0+0.8$ can be estimated:

$Q_{0}^{\prime}<\frac{R^{2}}{4}\left[\frac{1}{r_{1}^{2}} Q_{0}(\mathrm{O} 5 \mathrm{~V})+\frac{1}{r_{2}^{2}} Q_{0}(\mathrm{O} 6 \mathrm{~V})\right]$

where $r_{1}$ and $r_{2}$ are the distances to the $\mathrm{O} 5 \mathrm{~V}$ and $\mathrm{O} 6 \mathrm{~V}$ stars respectively and $Q_{0}(\mathrm{O} 5 \mathrm{~V})$ and $Q_{0}(\mathrm{O} 6 \mathrm{~V})$ are their ionizing fluxes, which we take from Schaerer \& de Koter (1997). The upper limit is due to our neglecting the ionizing photons that are used up in keeping the intervening column of gas between those stars and G84.0+0.8 ionized and their absorption by dust, and as by our use of the projected distances given above as lower limits for $r_{1}$ and $r_{2}$. Still, we obtain $Q_{0}^{\prime}=1.9 \times 10^{44} \mathrm{~s}^{-1}$, insufficient by a factor of at least 14 to fulfill the requirements set by the properties of the G84.0+0.8 HII region.

It is nevertheless doubtful that J205551.3+435225 and HD 199579 represent the whole census of O-type stars in that region of the sky, as warned by the fact that a highly obscured O5V star such as J205551.3+435225 itself was only very recently identified. This is even more so if G84.0+0.8 is farther than W80, as the characteristics of the nebula hint (Sect. 3.2). Combining Eqs. (1) and (3) (the latter for a single-star case) to estimate the distance $r$ at which the ionizing star should be found, and again neglecting the photons absorbed between the star and the nebula, one obtains

$r=\left(\frac{3 Q_{0}}{16 \pi \beta_{2} \theta D n^{2}}\right)^{1 / 2}$ 
where $\theta$ is the angular radius of the nebula. This quantity defines a useful search radius where the ionizing star should be located, corresponding to $22^{\prime} \times(0.6 \mathrm{kpc} / D)^{3 / 2}$. The identification of a suitable ionizing star in its proximity could validate this tentative scenario for the nature of $\mathrm{G} 84.0+0.8$ by providing a reliable determination of its distance.

\section{Conclusions}

G84.0+0.8 is a moderate-density $\left(n \simeq 460 \mathrm{~cm}^{-3}\right)$ compact HII region in the Cygnus arm ionized by mid O-type stars and containing a lightly embedded cluster. Trying to fit the emission-line spectrum, the radio-continuum flux and the characteristics of its embedded stars in the standard picture of a cluster with massive stars exciting a compact HII region leads us to conclude that there is a significant mismatch between the ionizing flux supplied by a mid O-type star and that inferred from the optical and radio properties of the nebula, regardless of its distance to the Sun. When a large distance is assumed the brightest stars in the color-magnitude diagram occupy the positions of the required mid O-type stars and the ionizing flux mismatch becomes smaller, although still exceeding one order of magnitude. Moreover, the mid-to-far infrared luminosity of G84.0+0.8 is distinctly less steep than those of compact HII regions containing O-type stars, which might mean that the cluster embedded in G84.0+0.8 lacks the hot stars required to clear their vicinity from hot dust. We consider as an alternative interpretation that G84.0+0.8 may be a cavity of low density gas carved by a low-mass cluster in its parental molecular cloud and exposed to the ambient ionizing radiation field. Although no O-type star that may externally ionize G84.0+0.8 is identified, this should not be used at this stage to reject this possibility, given the likely incomplete census of O-type stars in the region.

Although the combination of existing observations with the new ones presented here does not definitively establish or rule out the membership of G84.0+0.8 of the North America and Pelican nebulae complex, they do provide a conclusive negative answer to the question posed by the title of this paper. It may be indeed tempting to regard a compact HII region containing a compact embedded cluster dominated by a relatively bright star as a likely massive star forming site in W80. However, the available observations indicate that the brightest star at its position is a late G-type projected by chance on the cluster boundaries, that the required ionizing flux is much below that supplied by a star of the inferred spectral type, and that the cluster does not contain stars of sufficiently early spectral type if G84.0+0.8 is as nearby as the North America or the Pelican nebulae. Still, G84.0+0.8 stands out as an interesting object of study whose relatively simple geometry may lend itself to relatively easy modeling.

Acknowledgements. We are very pleased to thank the staff of the Calar Alto observatory, and especially Mr. Santos Pedraz, Mr. Alberto Aguirre, Ms. Ana Guijarro, and Mr. Jesús Aceituno, for the excellent support during our observing runs in Calar Alto. We also thank Dr. Roland Gredel, Director of the Calar Alto observatory, for the allocation of Director's Discretionary Time (DDT) to this project, and to Mr. Manuel Alises for the execution of the DDT observations in Service Mode. A.P. acknowledges support from the OPTICON Network. J.T. acknowledges support by the Spanish Ministry of Science and Technology under contract AYA2003-07736. This research has made use of the SIMBAD database operated at CDS, Strasbourg, France. It also makes use of data products from the Two Micron All Sky Survey, which is a joint project of the University of Massachusetts and the Infrared Processing and Analysis Center/California Institute of Technology, funded by the National Aeronautics and Space Administration and the National Science Foundation.

\section{References}

Altenhoff, W., Mezger, P. G., Wendker, H., \& Westerhout, G. 1960, Veröff. Sternw. Bonn, 59, 48

Bally, J., \& Reipurth, B. 2003, AJ, 126, 893

Behre, O. P., Wendker, H. J., Higgs, L. A., \& Landecker, T. L. 2004, A\&A, 415, 217

Cardelli, J. A., Clayton, G. C., \& Mathis, J. S. 1989, ApJ, 345, 245

Chini, R., Krügel, E., \& Wargau, W. 1987, A\&A, 181, 378

Comerón, F., \& Pasquali, A. 2005, A\&A, 430, 541

Comerón, F., \& Torra, J. 2001, A\&A, 375, 539

Drilling, J. S., \& Landolt, A. U. 2000, in Allen's Astrophysical Quantities, ed. A. N. Cox (AIP Press)

Dutra, C. M., \& Bica, E. 2001, A\&A, 376, 434

Faison, M., Churchwell, E., Hofner, P., et al. 1998, ApJ, 500, 280

Gregory, P. C., \& Taylor, A. R. 1986, AJ, 92, 371

Griffith, M., Heflin, M., Conner, S., Burke, B., \& Langston, G. 1991, ApJS, 75, 801

Herbig, G. H. 1958, ApJ, 128, 259

Herbig, G. H., \& Terndrup, D. M. 1986, ApJ, 307, 609

Inoue, A. K. 2002, ApJ, 570, 688

Jacoby, G. H., Hunter, D. A., \& Christian, C. A. 1984, ApJS, 56, 357

Laugalys, V., \& Strayzis, V. 2002, Balt. Astron., 11, 205

Leung, H. O., \& Thaddeus, P. 1992, ApJS, 81, 267

Mezger, P. G., \& Henderson, A. P. 1967, ApJ, 147, 471

Miller, G. E., \& Scalo, J. M. 1979, ApJS, 41, 513

Normandeau, M., Joncas, G., \& Green, D. A. 1992, A\&AS, 92, 63

Odenwald, S. F., \& Schwartz, P. R. 1993, ApJ, 405, 706

Osterbrock, D. E. 1989, Astrophysics of Gaseous Nebulae and Active Galactic Nuclei (University Science Books)

Piepenbrink, A., \& Wendker, H. J. 1988, A\&A, 191, 313

Rieke, G. H., \& Lebofsky, M. J. 1985, ApJ, 288, 618

Schaerer, D., \& de Koter, A. 1997, A\&A, 322, 598

Shaver, P. A., McGee, R. X., Newton, L. M., Danks, A. C., \& Pottasch, S. R. 1983, MNRAS, 204, 53

Stasińska, G., \& Schaerer, D. 1997, A\&A, 322, 615

Tokunaga, A. T., 2000, in Allen's Astrophysical Quantities, ed. A. N. Cox (AIP Press)

Vacca, W. D., Garmany, C. D., \& Shull, J. M. 1996, ApJ, 460, 914

Wendker, H. J., Higgs, L. A., \& Landecker, T. L. 1991, A\&A, 241, 551

Westerhout, G. 1958, Bull. Astron. Inst. Netherlands, 14, 215

Wood, D. O. S., \& Churchwell, E. 1989, ApJS, 69, 831 